\title{
A population-based study of the epidemiology and clinical features of methicillin-resistant Staphylococcus aureus infection in Pennsylvania, 2001-2010
}

\author{
J. A. CASEY ${ }^{1}$, S. E. COSGROVE ${ }^{2,4}$, W. F. STEWART ${ }^{2,5}$, J. POLLAK $^{1}$ \\ AND B.S. SCHWARTZ $Z^{1,2,3,5 *}$ \\ ${ }^{1}$ Department of Environmental Health Sciences and ${ }^{2}$ Department of Epidemiology, Johns Hopkins Bloomberg \\ School of Public Health, Baltimore, MD, USA \\ ${ }^{3}$ Department of Medicine and ${ }^{4}$ Division of Infectious Disease, Johns Hopkins School of Medicine, Baltimore, \\ $M D, U S A$ \\ ${ }^{5}$ Center for Health Research, Geisinger Health System, Danville, PA, USA
}

Received 20 June 2012; Final revision 30 July 2012; Accepted 31 July 2012;

first published online 29 August 2012

\section{SUMMARY}

No U.S. general population-based study has characterized the epidemiology and risk factors, including skin and soft tissue infection (SSTI), for healthcare-associated (HA) and communityassociated (CA) methicillin-resistant Staphylococcus aureus (MRSA). We estimated the incidence of HA- and CA-MRSA and SSTI over a 9-year period using electronic health record data from the Geisinger Clinic in Pennsylvania. MRSA cases were frequency-matched to SSTI cases and controls in a nested case-control analysis. Logistic regression was used to assess risk factors, while accounting for antibiotic administration. We identified 1713 incident CA- and 1506 HA-MRSA cases and 78216 SSTI cases. On average, from 2005 to 2009, the annual incidence of CA-MRSA increased by $34 \%$, HA-MRSA by $7 \%$, and SSTI by $4 \%$. Age, season, community socioeconomic deprivation, obesity, smoking, previous SSTI, and antibiotic administration were identified as independent risk factors for CA-MRSA.

Key words: Epidemiology, incidence, methicillin-resistant S. aureus (MRSA), skin infections.

\section{INTRODUCTION}

Until the late 1990s methicillin-resistant Staphylococcus aureus (MRSA) was primarily a healthcareassociated (HA) pathogen. Thereafter, in the USA, younger, healthier individuals with none or few traditional healthcare risk factors began acquiring community-associated MRSA (CA-MRSA), which often presents as skin and soft tissue infections

\footnotetext{
* Author for correspondence: Dr B. S. Schwartz, Department of Environmental Health Sciences, Johns Hopkins Bloomberg School of Public Health, 615 N. Wolfe Street, Room W7041, Baltimore, MD, 21205, USA.

(Email: bschwart@jhsph.edu)
}

(SSTIs) [1, 2]. Data describing trends in the annual incidence of and the risk factors for HA- and CA-MRSA in a general population sample over the past decade are not available. Limitations in existing US research include study of: only invasive infection [3-7]; restricted populations (e.g. military, inmate, athlete) [6-16]; patients from hospital-based surveillance that could bias ascertainment towards HArather than CA-MRSA [16-19]; and limited time windows $[16,20]$. Among those studies, only two $[11,20]$ applied rigorous case definitions to separate CA- and HA-MRSA; others used location of onset as a surrogate or categorized using antibiotic-resistance profiles. Furthermore, trends in SSTI incidence in the 
general population are important because these infections could represent a source of undiagnosed MRSA, but have not been well described. No previous studies have included both MRSA and a range of SSTIs.

Since no longitudinal U.S. population-based study has been conducted including both HA- and CA-MRSA for all clinical indications in a large contiguous geography, little is known about healthcare and non-healthcare risk factors for HA- and CAMRSA and SSTIs in the general US population. We characterized the epidemiology of MRSA using 10 years of medical data from the electronic health record (EHR) of the Geisinger Health System in Pennsylvania. The EHR includes data on over 440000 primary-care patients of all ages that live in both urban and rural areas. We evaluated individual, community, and clinical risk factors for CA- and HA-MRSA patients and SSTI patients, compared to controls. Our goals were to: (1) estimate the incidence of HA- and CA-MRSA and SSTIs over a 10-year period; and (2) examine clinical and non-healthcare risk factors for HA- and CA-MRSA and SSTIs, with and without consideration of antibiotic use.

\section{METHODS}

\section{Study overview}

We first determined MRSA and SSTI incidence rates from 2001 to 2009 (in incident cases per 100000 person-years). Any patient with an inpatient or outpatient encounter in a given calendar year contributed 1 person-year to the denominator. We next selected cases and controls to perform a nested case-control analysis. The study was approved by Institutional Review Boards at the Johns Hopkins Bloomberg School of Public Health and the Geisinger Health System.

\section{Study population and design}

Data were obtained on 446480 patients with a Geisinger Clinic primary-care provider, from outpatient encounter records from 1 January 2001 to 9 February 2010 and from inpatient encounter records from 1 July 2003 to 9 February 2010. The system provides primary-care services in 41 community practice clinics and four hospitals in a 31-county region of central and northeastern Pennsylvania. We utilized only the primary-care population because health data are more complete and because it is representative of the general population in the region. Patients were geocoded using ArcGIS as described previously [21].

\section{Data sources}

Data in 157106929 records consisted of demographics, inpatient, outpatient, and emergency department encounters, laboratory data, medication orders, and procedures. All orders and encounters were accompanied by International Classification of Diseases, 9th Revision, Clinical Modification (ICD-9) codes or Geisinger system (EP) codes for diagnoses, and Current Procedural Terminology (CPT) codes for encounters, laboratory tests and procedures. Specific ICD-9 codes for MRSA only became available after 2007, but the Geisinger Clinic began using a system code for MRSA (EP884) in 2003.

\section{Identification of MRSA cases, SSTI cases and controls}

Incident MRSA was defined as: (1) a culture positive for MRSA; (2) an ICD-9 or EP code for MRSA; or (3) an ICD-9 code for $S$. aureus infection with an ICD-9 code for penicillin resistance (the clinical microbiology laboratory method to encode MRSA before 2007) (Fig. 1). The year of onset was defined as the first diagnosis of MRSA infection. No recurrent MRSA infections were included. We identified community-onset SSTI (CO-SSTI) cases (using 29 ICD-9 codes) using outpatient records; an incident CO-SSTI was defined as the first occurrence in any 6-month period. For the case-control analysis, SSTIs who never had a MRSA diagnosis were randomly selected and frequency-matched to the MRSA cases on age, sex, and year of diagnosis. Controls were also randomly selected and frequency-matched to MRSA cases on age, sex and an outpatient encounter in the same year as MRSA diagnosis. If SSTI cases or controls had multiple SSTI diagnoses or visits, respectively, during the matched year of MRSA diagnosis a single visit was randomly selected as the match visit.

\section{Assignment to HA- or CA-MRSA}

Assignment required inpatient records from the year preceding diagnosis; so all cases diagnosed before 2005 were excluded from this categorization $(n=854$, primarily hospital-onset MRSA). MRSA 


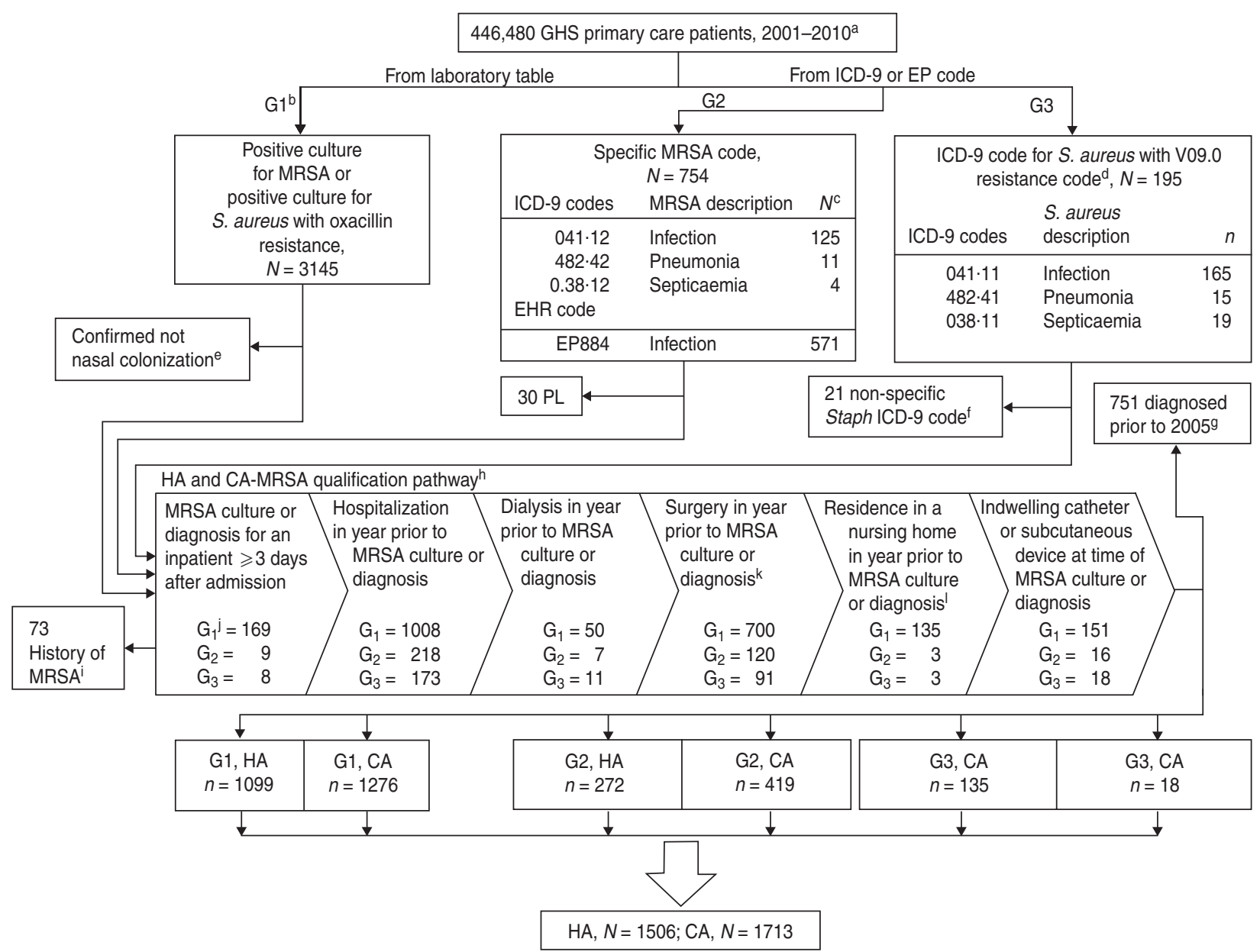

Fig. 1. Flow chart depicting MRSA case selection and diagnosis location. GHS, Geisinger Health System; G1, Group 1; G2, Group 2; G3, Group 3; ICD-9, International Classification of Diseases, 9th Revision, Clinical Modification; EP, Geisinger Clinic generated electronic health record code; PL, problem list table; HA-MRSA, healthcare-associated methicillinresistant Staphylococcus aureus; CA-MRSA, community associated MRSA; S. aureus, Staphylococcus aureus. ${ }^{\text {a }}$ Geisinger Health System, outpatient data was available 2001-2010, and inpatient data 2004-2010. ${ }^{\mathrm{b}} \mathrm{G} 1, \mathrm{G} 2$ and G3 indicate the method by which MRSA cases were identified. The numbered hierarchy was used when a case was identified by multiple methods on the same day, otherwise the case was linked to the method that identified the earliest MRSA diagnosis. ${ }^{\mathrm{c}}$ These numbers are not mutually exclusive. ${ }^{\mathrm{d}}$ Before 2007 there was no MRSA-specific ICD-9 code and MRSA was identified at GHS by the analogous MSSA code plus a V09.0 code, indicating infection with microorganisms resistant to penicillins. ${ }^{\mathrm{e}}$ Checked for PCR indicating MRSA colonization. ${ }^{\mathrm{f}}$ Cases originally selected with codes $041 \cdot 10$ (Staphylococcus infection, unspecified) and 038.10 (Staphylococcus septicaemia, unspecified) were excluded. ${ }^{\mathrm{g}}$ Due to the lack of inpatient data before 2004, cases could not be categorized as CA- or HA-MRSA before 2005. ${ }^{\mathrm{h}}$ If a patient met any of the six criteria they were classified as HA-MRSA. ${ }^{i} \mathrm{~V} 12.04$, the ICD-9 code for history of MRSA infection in record. ${ }^{j}$ Patients were disqualified from the CA-MRSA category if their procedures file contained a surgery or their inpatient, outpatient or emergency department file contained a post-operative visit in the 330 days before MRSA infection. ${ }^{\mathrm{k}} n_{1}, n_{2}, n_{3}$ correspond to the numbered hierarchy and represent the total number of patients with each characteristic by group (i.e. these numbers are not mutually exclusive). ${ }^{1}$ Patients were disqualified from the CA-MRSA category if their address matched an address of a nursing home facility in Pennsylvania listed on the Nursing Home Compare database website provided by the U.S. Department of Health and Human Services.

was classified as CA based on the case definition of the Centers for Disease Control and Prevention (CDC); all other cases were categorized as HA $[20,22]$. Antibiotic susceptibility of isolates and the site of infection were assessed in the subset of cases identified by culture.

\section{Statistical analysis}

Using data from 2005 to 2010, HA-MRSA, CAMRSA, and SSTI cases were compared to controls on demographics, body mass index (BMI), season of infection, community socioeconomic deprivation 
(CSD), residential community (representing the macro-environment of the patient, defined by census tracts in cities, moderate to high density boroughs, and suburban and rural townships), SSTI diagnosis in varying time windows in the year before MRSA diagnosis, antibiotic prescriptions, and selected acute and chronic health conditions [23]. We determined antibiotic administration in the 30-365 days preceding diagnosis to avoid protopathic bias [24].

Multinomial logistic regression was used to compare HA- and CA-MRSA cases to controls and a separate logistic regression model was used to compare SSTI cases to controls. We first present crude odds ratios (ORs) [with 95\% confidence intervals (CIs)]. We then adjusted for the following potential confounders: age (grouped to balance sample size and life stage considerations), sex, race/ethnicity and smoking status (ever vs. never). Health condition ORs were determined with and without adjustment for any antibiotic administration in the previous 2 years. We also assessed associations for antibiotic administration for specific indications and subsequent MRSA or SSTI infection.

All analysis was performed using Stata version 11.2 (StataCorp, USA) and R version 12.2.2 software (www.r-project.org). A multilevel multinomial logistic model (Stata gllamm with random intercept) was used to assess the association between case status and residential communities, as well as CSD [23].

\section{RESULTS}

\section{Incidence of MRSA infection and SSTIs}

From 2001-2009 an annual mean of 211102 (S.D. $=29301)$ unique patients had an inpatient or outpatient encounter. A total of 4094 MRSA cases and 78216 SSTI cases were identified between 1 January 2001 and 9 February 2010. After 1 January 2005, we were able to categorize 1506 as HA- and 1713 as CA-MRSA cases. MRSA cases, selected SSTI cases and controls had a mean of $38($ s.D. $=32)$ outpatient visits over the follow-up period and $6($ s.D. $=3$ ) years of follow-up time between their first and last outpatient encounter in the EHR, providing strong evidence that these patients were longitudinally followed. The mean annual incidence rate from 2001-2009 for all MRSA cases was 195 (95\% CI 189-201) cases $/ 100000$ person-years, compared to 4008 (95\% CI 3980-4037) SSTI cases/100 000 personyears. The mean percent annual increase in MRSA

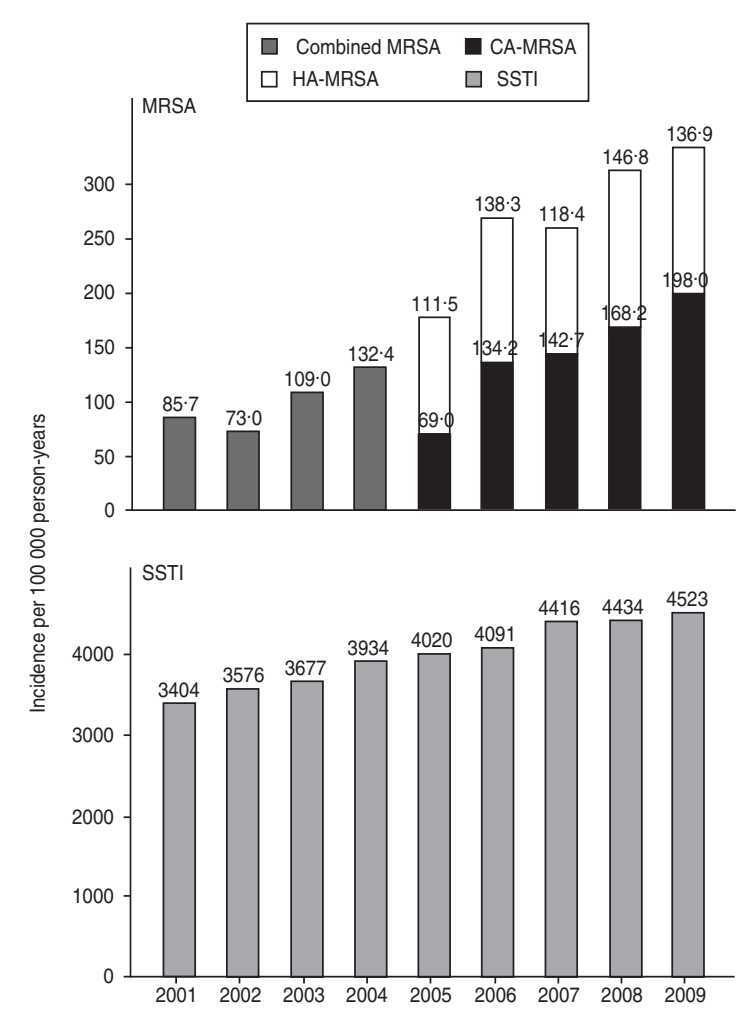

Fig. 2. Incidence of MRSA infection and SSTI per 100000 person-years among the Geisinger Clinic's primary-care patients, 2001-2009 (MRSA cases are combined before 2005 because lack of inpatient data did not allow for assignment to HA- and CA-MRSA groups). CA-MRSA, Community-associated MRSA; HA-MRSA, healthcareassociated MRSA; SSTI, skin and soft tissue infection.

incidence over the 9 years was $23 \%$ (range $-15 \%$ to $51 \%$ ), compared to $3.6 \%$ (range 1-8\%) for SSTI cases. The average annual percent increase in HAMRSA incidence was $7 \%$ (range $-13 \%$ to $23 \%$ ). In contrast, the average annual increase in CA-MRSA incidence was 34\% (range 6-94\%) from 2005 to 2009, with the largest percent and absolute increase during 2005-2006 (Fig. 2). For CA-MRSA, no rate exceeded 100/100000 person-years in patients aged $>46$ years, but several age- and sex-specific rates exceeded 300/100000 person-years including girls aged $<14$ years, the group that saw the most impressive increases incidence. SSTI incidence increased reasonably monotonically as a whole and in age and sex subgroups. The percent of total MRSA cases classified as CA rose from $38 \%$ in 2005 to $59 \%$ in 2009.

\section{Demographic and clinical comparisons of HA-MRSA, CA-MRSA, SSTIs and controls}

The majority ( $77.5 \%$ ) of MRSA cases were identified by laboratory culture (many also had ICD-9 codes), 
followed by specific ICD-9 codes only (17.6\%) and combined ICD-9 codes only (4.9\%). Patient characteristics did not differ by method of identification (data not shown), and a subgroup analysis using only cases with both a positive culture and a MRSA ICD-9 code on the same day $(n=824)$ did not reveal any substantive change in associations; therefore, all MRSA cases were combined in subsequent analyses. Among SSTI cases the three most common diagnoses were cellulitis $(62 \%)$, carbuncle $(17 \%)$, and impetigo (12\%). Only $8.5 \%$ of SSTI cases had a culture taken, and $S$. aureus was by far the most common organism identified, followed by Pseudomonas aeruginosa and Escherichia coli.

HA-MRSA cases were substantially older and less likely to be a race/ethnic minority than CA-MRSA cases (Table 1). For each identification method, HA cases had more outpatient encounters per year, compared to CA cases, SSTI cases and controls. HA isolates had higher odds of being resistant to two or more antibiotic classes than CA isolates (OR 6.47, $95 \%$ CI $4 \cdot 92-8 \cdot 60)$. Specimen descriptions were available for 954 HA- and 1088 CA-MRSA cases; of these the most common sources for HA-MRSA were skin and soft tissue $(50 \cdot 3 \%)$, respiratory $(16 \cdot 0 \%)$, urine $(15.9 \%)$ and blood $(8.0 \%)$, and for CA-MRSA were skin and soft tissue $(86 \cdot 4 \%)$, other $(7 \cdot 1 \%)$, urine $(5 \cdot 2 \%)$ and respiratory $(0 \cdot 6 \%)$.

\section{Associations of non-healthcare risk factors with case status}

Obesity, ever smoking and summer and autumn seasons were associated in both unadjusted and adjusted analyses with HA- and CA-MRSA and SSTI compared to controls (Table 2). There were no substantive changes in point estimates when CSD was added to the model (data not shown). An urban living environment was associated with both HA- and CA-MRSA, but not with SSTI. More deprived places (i.e. higher CSD) were also associated with higher odds of HA- and CA-MRSA (OR 1.2 and $1 \cdot 1$ per quartile, respectively), but not SSTI.

\section{Associations of antibiotic administration with case status}

MRSA and SSTI cases were significantly more likely than controls to have an antibiotic order in the 2 years before diagnosis (Table 1). Four or more antibiotic orders was associated with nearly a tenfold increase in the odds of HA-MRSA and a fourfold increase in the odds of CA-MRSA compared to those who received no antibiotics (Table 2). In particular, the more antibiotics prescribed in the 30-365 days before diagnosis of CA-MRSA, the higher the odds of CA-MRSA in both unadjusted and adjusted analyses. Most classes of antibiotics commonly prescribed in the outpatient setting, with the exception of macrolides, were independently associated with increased risk of CAMRSA. The associations between antibiotic prescribing and CA-MRSA remained when antibiotic prescriptions were assessed in the 90-365 days before diagnosis, suggesting the association is not only due to protopathic bias from antibiotics for undiagnosed CA-MRSA infection (data not shown).

\section{Associations of previous SSTIs with case status}

On the day of diagnosis, the majority of CA-MRSA cases presented with a SSTI $(n=1028,60 \%)$, compared to a quarter of HA-MRSA cases $(n=429)$, all SSTI cases (by definition) and less than $2 \%$ of controls. In the year before MRSA or SSTI diagnosis or visit date, SSTI diagnosis in all time windows was strongly associated with case status (Fig. 3). Previous SSTI was a risk factor for subsequent SSTI with a linear decline in odds as time from diagnosis increased. In contrast, the associations of previous SSTI with subsequent diagnosis of both HA and CA-MRSA were nonlinear and stronger than these were for subsequent diagnosis of SSTI.

\section{Associations of selected healthcare risk factors with case status}

In unadjusted analysis, in the 2 years preceding diagnosis, a visit for most respiratory tract, cardiac, and skin conditions was associated with HA- and CA-MRSA and SSTI case status (Table 3). After adjustment for antibiotic administration, only SSTI visit remained associated in all three comparisons. Asthma and chronic obstructive pulmonary disease (COPD) remained associated with both HA-MRSA and SSTI after adjustment. Hypertension, diabetes, heart disease, and kidney disease were each risk factors for HA-MRSA and SSTIs, but not for CA-MRSA, suggesting that CA-MRSA cases were healthier than HA-MRSA cases, SSTIs and controls. In adjusted analysis, antibiotic orders in the previous 2 years for several acute respiratory tract conditions and SSTIs were strongly associated with MRSA and 
Table 1. Patient demographic and clinical characteristics

\begin{tabular}{|c|c|c|c|c|}
\hline Characteristic & $\begin{array}{l}\text { HA-MRSA } \\
(n=1506)\end{array}$ & $\begin{array}{l}\text { CA-MRSA } \\
(n=1713)\end{array}$ & $\begin{array}{l}\text { SSTI } \\
(n=3336)\end{array}$ & $\begin{array}{l}\text { Control } \\
(n=3336)\end{array}$ \\
\hline Male sex & $766(51)$ & $803(47)$ & $1626(49)$ & $1626(49)$ \\
\hline \multicolumn{5}{|l|}{ Age in years at infection or visit } \\
\hline Median & $61 \cdot 1$ & $24 \cdot 0$ & $42 \cdot 0$ & $42 \cdot 0$ \\
\hline$<7$ & $139(9)$ & $319(19)$ & $475(14)$ & $475(14)$ \\
\hline 7 to $<19$ & $72(5)$ & $407(24)$ & $495(15)$ & $497(15)$ \\
\hline 19 to $<46$ & $246(16)$ & $545(32)$ & $820(25)$ & $816(25)$ \\
\hline 46 to $<62$ & $313(21)$ & $247(14)$ & $583(18)$ & $583(18)$ \\
\hline 62 to $<75$ & $289(19)$ & $111(6)$ & $423(13)$ & $487(14)$ \\
\hline$\geqslant 75$ & $447(30)$ & $84(5)$ & $540(16)$ & $498(15)$ \\
\hline \multicolumn{5}{|l|}{ Race/ethnicity } \\
\hline White $^{\mathrm{a}}$ & $1463(96)$ & $1611(94)$ & $3199(96)$ & $3165(95)$ \\
\hline Black & $28(2)$ & $53(3)$ & $51(2)$ & $64(2)$ \\
\hline Hispanic & $15(1)$ & $35(2)$ & $59(2)$ & $64(2)$ \\
\hline Other & $11(1)$ & $13(1)$ & $27(1)$ & $42(1)$ \\
\hline Unknown & $4(0)$ & $1(0)$ & 0 & $3(0)$ \\
\hline Adult $\mathrm{BMI}^{\mathrm{b}}$ & $(n=1306)$ & $(n=1023)$ & $(n=2418)$ & $(n=2418)$ \\
\hline Normal & $329(25)$ & $222(22)$ & $601(25)$ & $523(22)$ \\
\hline Overweight & $284(22)$ & $247(24)$ & $1012(42)$ & $697(29)$ \\
\hline Obese & $527(40)$ & $398(39)$ & $372(15)$ & $819(34)$ \\
\hline Missing & $167(13)$ & $156(15)$ & $372(15)$ & $379(16)$ \\
\hline Child BMI ${ }^{\mathrm{c}}$ & $(n=101)$ & $(n=578)$ & $(n=805)$ & $(n=918)$ \\
\hline Normal & $49(49)$ & $236(41)$ & $352(44)$ & $488(53)$ \\
\hline Overweight & $14(14)$ & 109 (19) & $128(16)$ & $107(12)$ \\
\hline Obese & $28(28)$ & $103(18)$ & $124(15)$ & $95(10)$ \\
\hline Missing & $10(10)$ & $130(23)$ & $201(25)$ & $228(25)$ \\
\hline \multicolumn{5}{|l|}{ Smoking $^{\mathrm{d}}$} \\
\hline Never & $1075(71)$ & $1426(83)$ & $1540(90)$ & $3025(91)$ \\
\hline \multicolumn{5}{|l|}{ Season ${ }^{\mathrm{e}}$} \\
\hline Winter & $371(24)$ & $384(22)$ & $769(23)$ & $891(27)$ \\
\hline Spring & $338(22)$ & $306(18)$ & $753(23)$ & $815(24)$ \\
\hline Summer & $397(26)$ & $462(27)$ & $953(29)$ & $783(24)$ \\
\hline Autumn & $415(27)$ & $561(33)$ & $861(26)$ & $847(25)$ \\
\hline \multicolumn{5}{|l|}{ Community type $\mathrm{f}^{\mathrm{f}}$} \\
\hline City & $240(16)$ & $255(15)$ & $376(11)$ & $327(10)$ \\
\hline Borough & $386(26)$ & $542(32)$ & $840(25)$ & $854(26)$ \\
\hline Township & $700(46)$ & $732(43)$ & $1774(53)$ & $1802(55)$ \\
\hline Missing ${ }^{g}$ & $180(12)$ & $183(11)$ & $414(14)$ & $353(11)$ \\
\hline \multicolumn{5}{|l|}{ Socioeconomic deprivation ${ }^{\mathrm{h}}$} \\
\hline Median & $-4 \cdot 1$ & $-4 \cdot 3$ & $-5 \cdot 0$ & $-5 \cdot 0$ \\
\hline Any antibiotic prescription in previous 2 years & $1176(76)$ & $1246(73)$ & $2341(70)$ & $1720(52)$ \\
\hline \multicolumn{5}{|l|}{ Antibacterial prescriptions ${ }^{\mathrm{i}}$} \\
\hline No prescription & $582(39)$ & $810(47)$ & $1712(51)$ & $2228(67)$ \\
\hline Carbapenems & $8(1)$ & 0 & $2(<1)$ & 0 \\
\hline Cephalosporins & $394(26)$ & $315(18)$ & $502(15)$ & $220(7)$ \\
\hline Clindaymycin & $67(4)$ & $34(2)$ & $64(2)$ & $20(1)$ \\
\hline Linezolid & $7(<1)$ & 0 & $1(<1)$ & $1(<1)$ \\
\hline Macrolides & $220(14)$ & $237(14)$ & $460(14)$ & $215(9)$ \\
\hline Penicillins & $358(24)$ & $442(26)$ & $741(22)$ & $531(16)$ \\
\hline Penicillin & $12(1)$ & $12(1)$ & $26(1)$ & $22(1)$ \\
\hline Amino & $216(14)$ & $347(20)$ & $553(17)$ & $404(12)$ \\
\hline Anti-staphylococcal & $8(1)$ & $2(<1)$ & $4(<1)$ & $2(<1)$ \\
\hline$\beta$-lactam $/ \beta$-lactamase inhibitors & $176(12)$ & $159(9)$ & $267(8)$ & $153(5)$ \\
\hline
\end{tabular}


Table 1 (cont.)

\begin{tabular}{lllll}
\hline \hline Characteristic & $\begin{array}{l}\text { HA-MRSA } \\
(n=1506)\end{array}$ & $\begin{array}{l}\text { CA-MRSA } \\
(n=1713)\end{array}$ & $\begin{array}{l}\text { SSTI } \\
(n=3336)\end{array}$ & $\begin{array}{l}\text { Control } \\
(n=3336)\end{array}$ \\
\hline $\begin{array}{l}\text { Quinolones } \\
\text { Tetracyclines }\end{array}$ & $337(22)$ & $112(7)$ & $242(7)$ & $167(5)$ \\
TMP/SMX & $85(6)$ & $79(5)$ & $157(5)$ & $84(3)$ \\
$\quad \begin{array}{l}\text { Vancomycin } \\
\text { Antibacterial drug prescriptions }\end{array}$ & $171(11)$ & $174(10)$ & $225(7)$ & $136(4)$ \\
0 & $73(5)$ & $2(<1)$ & $19(1)$ & $9(<1)$ \\
1 & & & & \\
$2-3$ & $582(39)$ & $810(47)$ & $1705(51)$ & $2228(67)$ \\
$\geqslant 4$ & $289(19)$ & $396(23)$ & $783(23)$ & $639(19)$ \\
\hline \hline
\end{tabular}

Data are no. (\%) of patients, unless otherwise indicated.

BMI, Body mass index; CA-MRSA, community-associated methicillin-resistant S. aureus; HA-MRSA, healthcareassociated MRSA; SSTI, skin and soft tissue infection; TMP/SMX, trimethoprim/sulfamethoxzole.

${ }^{a}$ White, non-Hispanic.

b Body mass index was categorized as normal $\left(<25 \mathrm{~kg} / \mathrm{m}^{2}\right)$, overweight $\left(25-29 \cdot 9 \mathrm{~kg} / \mathrm{m}^{2}\right)$ and obese $\left(\geqslant 30 \mathrm{~kg} / \mathrm{m}^{2}\right)$ for persons aged 18-59.9 years the most recent height and a weight within 2 years of the encounter/visit were used; for persons aged $\geqslant 60$ years the most recent height and weight within 1 year of encounter/visit were used. Missing either due to the absolute value of the $z$ score being $>5$ or if a height and weight were not recorded in the vitals table within the 3 months before the diagnosis or visit.

${ }^{c}$ Body mass index $z$ scores for children aged 2-18 years were calculated using the 2000 CDC Growth Reference by implementing the zanthro function in Stata version 11 (normal, $z$ score $<85$ th percentile; overweight, 85 th percentile $\leqslant z$ score $<95$ th percentile; obese, $z$ score $\geqslant 95$ th percentile).

d Based on presence of ICD-9 codes 305.1 (tobacco use disorder), V15.82 (history of tobacco use), 649.0 (tobacco use complicating pregnancy) or CPT codes 99406 or 99407 (smoking cessation counselling).

e Season of onset: spring (March-May), summer (June-August), autumn (September-November), winter (DecemberFebruary).

${ }^{\mathrm{f}}$ Census tracts were assigned to patients in cities due to the large geographical area and heterogeneous community of some cities.

g The overall geocoding rate was $88.6 \%$, non-geocoding patients could not be assigned a community type or a community socioeconomic deprivation score and thus were omitted from multilevel analysis.

${ }^{\mathrm{h}}$ Community socioeconomic deprivation was assigned at the township, borough or census tract-level and is based on six indicators (all percentages) derived from US Census 2000 data: combined less than high school education, not in the labour force, in poverty, on public assistance, civilian unemployment, and does not own a car; a higher score represents a more deprived community.

i Order for an antimicrobial prescription in the 30-365 days before infection or visit.

${ }^{j}$ Count of antimicrobial prescription order in the 30-365 days before infection or visit.

SSTI (Table 4). The number of previous healthcare visits also increased the odds of HA (OR 1 10, 95\% CI $1 \cdot 10-1 \cdot 12)$ and, to a lesser extent, CA-MRSA (OR $1 \cdot 01,95 \%$ CI $1 \cdot 0-1 \cdot 02)$.

\section{DISCUSSION}

This study revealed a substantial increase in CAMRSA, a stabilization of HA-MRSA, and a modest increase in SSTI incidence over a 10-year period in a large general population-based sample in the USA. The 2009 CA-MRSA incidence of 198/100000 person-years in our combined urban and rural cohort is similar to previous estimates, which were derived mainly from urban areas $[8,11,15,16,25]$, suggesting that the incidence CA-MRSA in rural areas may be greater than currently appreciated. The incidence of CO-SSTI was consistently 25 times higher than that of CA-MRSA. Previous work on MRSA infection has not included a SSTI comparison group and has generally used only one method to identify MRSA cases, while we used two methods to enhance case detection $[8,10,14,16,17,25-29]$. We confirmed previous reported, and identified new risk factors for CA-MRSA. Obesity, smoking and use of antibiotics conferred particular risk. The finding that SSTI as long as 1 year before was an independent risk factor for subsequent MRSA diagnosis suggests either that 
Table 2. Associations of demographic and clinical characteristics with MRSA and SSTI case status compared to controls, in multinomial and binomial logistic regression models, respectively ${ }^{\mathrm{a}}$

\begin{tabular}{|c|c|c|c|c|c|c|}
\hline \multirow[b]{2}{*}{ Characteristic } & \multicolumn{2}{|l|}{ HA-MRSA } & \multicolumn{2}{|l|}{ CA-MRSA } & \multicolumn{2}{|l|}{ SSTI } \\
\hline & $\begin{array}{l}\text { Crude OR } \\
(95 \% \mathrm{CI})\end{array}$ & $\begin{array}{l}\text { Adjusted } \mathrm{OR}^{\mathrm{b}} \\
(95 \% \mathrm{CI})\end{array}$ & $\begin{array}{l}\text { Crude OR } \\
(95 \% \mathrm{CI})\end{array}$ & $\begin{array}{l}\text { Adjusted OR } \\
(95 \% \mathrm{CI})\end{array}$ & $\begin{array}{l}\text { Crude OR } \\
(95 \% \mathrm{CI})\end{array}$ & $\begin{array}{l}\text { Adjusted OR } \\
(95 \% \mathrm{CI})\end{array}$ \\
\hline Male sex & $1 \cdot 1(1 \cdot 0-1 \cdot 2)$ & $1 \cdot 0(0 \cdot 9-1 \cdot 2)$ & $0.9(0 \cdot 8-1 \cdot 0)$ & $0 \cdot 9(0 \cdot 8-1 \cdot 0)$ & $1 \cdot 0(0 \cdot 9-1 \cdot 1)$ & $1 \cdot 0(0 \cdot 9-1 \cdot 1)$ \\
\hline $\begin{array}{l}\text { Age in years at infection or visit } \\
\qquad 7 \\
7 \text { to }<19 \\
19 \text { to }<46 \\
46 \text { to }<62 \\
62 \text { to }<75 \\
\geqslant 75\end{array}$ & $\begin{array}{l}0.5(0.4-0.7) \\
0.3(0.2-0.4) \\
0.6(0.5-0.7) \\
\text { Reference } \\
1 \cdot 2(0.9-1 \cdot 4) \\
1 \cdot 7(1.4-2 \cdot 0)\end{array}$ & $\begin{array}{l}0 \cdot 8(0 \cdot 6-1 \cdot 0) \\
0 \cdot 4(0 \cdot 3-0 \cdot 5) \\
0 \cdot 6(0 \cdot 5-0 \cdot 7) \\
\text { Reference } \\
1 \cdot 2(1 \cdot 0-1 \cdot 5) \\
2 \cdot 0(1 \cdot 7-2 \cdot 4)\end{array}$ & $\begin{array}{l}1.6(1.3-1.9) \\
1.9(1.6-2.4) \\
1.6(1.3-1.9) \\
\text { Reference } \\
0.6(0.4-0.7) \\
0.4(0.3-0.5)\end{array}$ & $\begin{array}{l}1.9(1.6-2.4) \\
2 \cdot 3(1.9-2.8) \\
1.6(1.3-1.9) \\
\text { Reference } \\
0.6(0.4-0.7) \\
0.4(0.3-0.6)\end{array}$ & $\begin{array}{l}1 \cdot 0(0 \cdot 8-1 \cdot 2) \\
1 \cdot 0(0 \cdot 8-1 \cdot 2) \\
1 \cdot 0(0 \cdot 9-1 \cdot 2) \\
\text { Reference } \\
0.9(0 \cdot 8-1 \cdot 1) \\
1 \cdot 1(0 \cdot 9-1 \cdot 3)\end{array}$ & $\begin{array}{l}1 \cdot 3(1 \cdot 1-1 \cdot 5) \\
1 \cdot 2(1 \cdot 0-1 \cdot 5) \\
1 \cdot 0(0 \cdot 9-1 \cdot 2) \\
\text { Reference } \\
1 \cdot 0(0 \cdot 8-1 \cdot 2) \\
1 \cdot 2(1 \cdot 0-1 \cdot 5)\end{array}$ \\
\hline $\begin{array}{l}\text { Race/ethnicity } \\
\text { White }^{\mathrm{c}} \\
\text { Black } \\
\text { Hispanic } \\
\text { Other }\end{array}$ & $\begin{array}{l}\text { Reference } \\
0 \cdot 9(0 \cdot 6-1 \cdot 5) \\
0 \cdot 5(0 \cdot 3-0 \cdot 9) \\
0 \cdot 6(0 \cdot 3-1 \cdot 1)\end{array}$ & $\begin{array}{l}\text { Reference } \\
1 \cdot 4(0 \cdot 9-2 \cdot 2) \\
0 \cdot 8(0 \cdot 4-1 \cdot 4) \\
0 \cdot 9(0 \cdot 5-1 \cdot 8)\end{array}$ & $\begin{array}{l}\text { Reference } \\
1 \cdot 6(1 \cdot 1-2 \cdot 4) \\
1 \cdot 1(0 \cdot 7-1 \cdot 7) \\
0 \cdot 6(0 \cdot 3-1 \cdot 1)\end{array}$ & $\begin{array}{l}\text { Reference } \\
1 \cdot 3(0 \cdot 9-1 \cdot 8) \\
0 \cdot 8(0 \cdot 5-1 \cdot 3) \\
0 \cdot 5(0 \cdot 3-1 \cdot 0)\end{array}$ & $\begin{array}{l}\text { Reference } \\
0 \cdot 8(0 \cdot 5-1 \cdot 1) \\
0 \cdot 9(0 \cdot 7-1 \cdot 4) \\
0 \cdot 6(0 \cdot 4-1 \cdot 0)\end{array}$ & $\begin{array}{l}\text { Reference } \\
0 \cdot 8(0 \cdot 5-1 \cdot 1) \\
1 \cdot 0(0 \cdot 7-1 \cdot 4) \\
0 \cdot 7(0 \cdot 4-1 \cdot 1)\end{array}$ \\
\hline $\begin{array}{l}\text { Adult BMI }{ }^{\mathrm{d}} \\
\text { Normal } \\
\text { Overweight } \\
\text { Obese }\end{array}$ & $\begin{array}{l}\text { Reference } \\
0 \cdot 6(0 \cdot 5-0 \cdot 8) \\
1 \cdot 3(1 \cdot 1-1 \cdot 5)\end{array}$ & $\begin{array}{l}\text { Reference } \\
0 \cdot 6(0 \cdot 5-0 \cdot 8) \\
1 \cdot 1(0 \cdot 9-1 \cdot 3)\end{array}$ & $\begin{array}{l}\text { Reference } \\
0 \cdot 8(0 \cdot 6-0 \cdot 9) \\
1 \cdot 3(1 \cdot 1-1 \cdot 5)\end{array}$ & $\begin{array}{l}\text { Reference } \\
1 \cdot 0(0 \cdot 8-1 \cdot 2) \\
1 \cdot 3(1 \cdot 1-1 \cdot 6)\end{array}$ & $\begin{array}{l}\text { Reference } \\
1 \cdot 7(1 \cdot 2-2 \cdot 2) \\
1 \cdot 8(1 \cdot 3-2 \cdot 4)\end{array}$ & $\begin{array}{l}\text { Reference } \\
1 \cdot 5(1 \cdot 1-2 \cdot 1) \\
1 \cdot 7(1 \cdot 2-2 \cdot 3)\end{array}$ \\
\hline $\begin{array}{l}\text { Child } \mathrm{BMI}^{\mathrm{e}} \\
\text { Normal } \\
\text { Overweight } \\
\text { Obese }\end{array}$ & $\begin{array}{l}\text { Reference } \\
0 \cdot 8(0 \cdot 4-1 \cdot 4) \\
2 \cdot 2(1 \cdot 3-3 \cdot 6)\end{array}$ & $\begin{array}{l}\text { Reference } \\
1 \cdot 0(0 \cdot 5-1 \cdot 8) \\
2 \cdot 2(1 \cdot 3-3 \cdot 8)\end{array}$ & $\begin{array}{l}\text { Reference } \\
1 \cdot 4(1 \cdot 0-1 \cdot 9) \\
1 \cdot 5(1 \cdot 1-2 \cdot 0)\end{array}$ & $\begin{array}{l}\text { Reference } \\
1 \cdot 5(1 \cdot 1-2 \cdot 1) \\
1 \cdot 6(1 \cdot 2-2 \cdot 3)\end{array}$ & $\begin{array}{l}\text { Reference } \\
1 \cdot 2(0 \cdot 9-1 \cdot 6) \\
1 \cdot 4(1 \cdot 0-1 \cdot 8)\end{array}$ & $\begin{array}{l}\text { Reference } \\
1 \cdot 2(0 \cdot 9-1 \cdot 7) \\
1 \cdot 4(1 \cdot 0-1 \cdot 9)\end{array}$ \\
\hline $\begin{array}{l}\text { Smoking } \\
\text { Ever }\end{array}$ & $4 \cdot 0(3 \cdot 4-4 \cdot 7)$ & $3 \cdot 9(3 \cdot 3-3 \cdot 6)$ & $2 \cdot 0(1 \cdot 6-2 \cdot 3)$ & $2 \cdot 4(2 \cdot 0-2 \cdot 8)$ & $2 \cdot 4(2 \cdot 0-2 \cdot 7)$ & $2 \cdot 4(2 \cdot 2-3 \cdot 0)$ \\
\hline $\begin{array}{l}\text { Season }^{\mathrm{f}} \\
\text { Winter } \\
\text { Spring } \\
\text { Summer } \\
\text { Autumn }\end{array}$ & $\begin{array}{l}\text { Reference } \\
1 \cdot 0(0 \cdot 8-1 \cdot 2) \\
1 \cdot 2(1 \cdot 0-1 \cdot 4) \\
1 \cdot 2(1 \cdot 0-1 \cdot 4)\end{array}$ & $\begin{array}{l}\text { Reference } \\
1 \cdot 0(0 \cdot 8-1 \cdot 2) \\
1 \cdot 3(1 \cdot 1-1 \cdot 5) \\
1 \cdot 2(1 \cdot 0-1 \cdot 4)\end{array}$ & $\begin{array}{l}\text { Reference } \\
0 \cdot 9(0 \cdot 7-1 \cdot 0) \\
1 \cdot 4(1 \cdot 2-1 \cdot 6) \\
1 \cdot 5(1 \cdot 3-1 \cdot 8)\end{array}$ & $\begin{array}{l}\text { Reference } \\
0 \cdot 9(0 \cdot 7-1 \cdot 1) \\
1 \cdot 3(1 \cdot 1-1 \cdot 6) \\
1 \cdot 5(1 \cdot 3-1 \cdot 8)\end{array}$ & $\begin{array}{l}\text { Reference } \\
1 \cdot 1(0 \cdot 9-1 \cdot 2) \\
1 \cdot 4(1 \cdot 2-1 \cdot 6) \\
1 \cdot 2(1 \cdot 0-1 \cdot 3)\end{array}$ & $\begin{array}{l}\text { Reference } \\
1 \cdot 1(0 \cdot 9-1 \cdot 2) \\
1 \cdot 4(1 \cdot 2-1 \cdot 6) \\
1 \cdot 2(1 \cdot 0-1 \cdot 3)\end{array}$ \\
\hline $\begin{array}{l}\text { Community type }{ }^{\mathrm{g}} \\
\text { Township } \\
\text { Borough } \\
\text { City }^{\mathrm{h}}\end{array}$ & $\begin{array}{l}\text { Reference } \\
1 \cdot 2(1 \cdot 0-1 \cdot 4) \\
2 \cdot 0(1 \cdot 6-2 \cdot 5)\end{array}$ & $\begin{array}{l}\text { Reference } \\
1 \cdot 2(1 \cdot 0-4 \cdot 0) \\
2 \cdot 1(1 \cdot 6-2 \cdot 6)\end{array}$ & $\begin{array}{l}\text { Reference } \\
1 \cdot 6(1 \cdot 3-1 \cdot 9) \\
2 \cdot 1(1 \cdot 6-2 \cdot 6)\end{array}$ & $\begin{array}{l}\text { Reference } \\
1 \cdot 5(1 \cdot 2-1 \cdot 8) \\
1 \cdot 8(1 \cdot 4-2 \cdot 3)\end{array}$ & $\begin{array}{l}\text { Reference } \\
1 \cdot 1(1 \cdot 0-1 \cdot 2) \\
1 \cdot 2(1 \cdot 0-1 \cdot 5)\end{array}$ & $\begin{array}{l}\text { Reference } \\
1 \cdot 1(0 \cdot 9-1 \cdot 2) \\
1 \cdot 2(1 \cdot 0-1 \cdot 4)\end{array}$ \\
\hline $\begin{array}{l}\text { Socioeconomic deprivation, } \\
\text { per quartile }\end{array}$ & $1 \cdot 2(1 \cdot 1-1 \cdot 3)$ & $1 \cdot 2(1 \cdot 1-1 \cdot 3)$ & $1 \cdot 2(1 \cdot 1-1 \cdot 3)$ & $1 \cdot 1(1 \cdot 1-1 \cdot 2)$ & $1 \cdot 1(1 \cdot 0-1 \cdot 1)$ & $1 \cdot 1(1 \cdot 0-1 \cdot 1)$ \\
\hline $\begin{array}{l}\text { Any antibiotic prescription in } \\
\text { previous } 2 \text { years }\end{array}$ & $3 \cdot 0(2 \cdot 7-3 \cdot 5)$ & $2 \cdot 9(2 \cdot 6-3 \cdot 4)$ & $2 \cdot 5(2 \cdot 2-2 \cdot 8)$ & $2 \cdot 4(2 \cdot 1-2 \cdot 8)$ & $2 \cdot 2(2 \cdot 0-2 \cdot 4)$ & $2 \cdot 1(1 \cdot 9-2 \cdot 3)$ \\
\hline
\end{tabular}




\begin{tabular}{|c|c|c|c|c|c|c|}
\hline \multirow[b]{2}{*}{ Characteristic } & \multicolumn{2}{|l|}{ HA-MRSA } & \multicolumn{2}{|l|}{ CA-MRSA } & \multicolumn{2}{|l|}{ SSTI } \\
\hline & $\begin{array}{l}\text { Crude OR } \\
(95 \% \mathrm{CI})\end{array}$ & $\begin{array}{l}\text { Adjusted } \mathrm{OR}^{\mathrm{b}} \\
(95 \% \mathrm{CI})\end{array}$ & $\begin{array}{l}\text { Crude OR } \\
(95 \% \mathrm{CI})\end{array}$ & $\begin{array}{l}\text { Adjusted OR } \\
(95 \% \mathrm{CI})\end{array}$ & $\begin{array}{l}\text { Crude OR } \\
(95 \% \mathrm{CI})\end{array}$ & $\begin{array}{l}\text { Adjusted OR } \\
(95 \% \mathrm{CI})\end{array}$ \\
\hline \multicolumn{7}{|l|}{ Antibiotic prescription ${ }^{\mathrm{j}}$} \\
\hline No prescription & Reference & Reference & Reference & Reference & Reference & Reference \\
\hline Cephalosporins & $6 \cdot 9(5 \cdot 7-8 \cdot 3)$ & $3 \cdot 4(2 \cdot 8-4 \cdot 2)$ & $3 \cdot 9(3 \cdot 3-4 \cdot 8)$ & $2 \cdot 8(2 \cdot 3-3 \cdot 4)$ & $2 \cdot 9(2 \cdot 5-3 \cdot 5)$ & $2 \cdot 1(1 \cdot 8-2 \cdot 5)$ \\
\hline Clindamycin & $12 \cdot 8(7 \cdot 7-21 \cdot 3)$ & $4 \cdot 2(2 \cdot 4-7 \cdot 4)$ & $4 \cdot 7(2 \cdot 7-8 \cdot 2)$ & $2 \cdot 6(1 \cdot 4-4 \cdot 6)$ & $4 \cdot 2(2 \cdot 5-6 \cdot 9)$ & $2 \cdot 3(1.4-3.9)$ \\
\hline Macrolides & $2 \cdot 7(2 \cdot 2-3 \cdot 3)$ & $1 \cdot 1(0 \cdot 9-1 \cdot 4)$ & $2 \cdot 1(1 \cdot 7-2 \cdot 5)$ & $1 \cdot 2(1 \cdot 0-1 \cdot 4)$ & $1 \cdot 9(1 \cdot 6-2 \cdot 2)$ & $1 \cdot 2(1 \cdot 0-1 \cdot 4)$ \\
\hline Penicillins & $2 \cdot 6(2 \cdot 3-3 \cdot 0)$ & $1.4(1 \cdot 2-1 \cdot 7)$ & $2 \cdot 3(2 \cdot 0-2 \cdot 7)$ & $1 \cdot 3(1 \cdot 1-1 \cdot 5)$ & $1 \cdot 8(1 \cdot 6-2 \cdot 1)$ & $1 \cdot 4(1 \cdot 2-1 \cdot 6)$ \\
\hline Penicillin & $2 \cdot 1(1 \cdot 0-4 \cdot 2)$ & $1 \cdot 8(0 \cdot 8-3 \cdot 9)$ & $1 \cdot 5(0 \cdot 8-3 \cdot 0)$ & $0 \cdot 7(0 \cdot 3-1 \cdot 4)$ & $1 \cdot 6(0 \cdot 9-2 \cdot 9)$ & $0.9(0 \cdot 5-1 \cdot 7)$ \\
\hline Amino & $2 \cdot 0(1 \cdot 7-2 \cdot 5)$ & $1 \cdot 2(1 \cdot 0-1 \cdot 5)$ & $2 \cdot 4(2 \cdot 0-2 \cdot 8)$ & $1 \cdot 3(1 \cdot 1-1 \cdot 6)$ & $1 \cdot 8(1 \cdot 5-2 \cdot 0)$ & $1 \cdot 3(1 \cdot 1-1 \cdot 5)$ \\
\hline Antistaphylococcal & $15 \cdot 3(3 \cdot 4-72 \cdot 4)$ & $3 \cdot 2(0 \cdot 6-18 \cdot 6)$ & $2 \cdot 8(0 \cdot 4-19 \cdot 6)$ & $1 \cdot 1(0 \cdot 1-8 \cdot 8)$ & $2 \cdot 6(0 \cdot 5-14 \cdot 2)$ & $1 \cdot 1(0 \cdot 2-7 \cdot 0)$ \\
\hline$\beta$-lactam $/ \beta$-lactamase inhibitors & $4 \cdot 4(3 \cdot 5-5 \cdot 6)$ & $1 \cdot 7(1 \cdot 3-2 \cdot 2)$ & $2 \cdot 9(2 \cdot 3-3 \cdot 6)$ & $1 \cdot 3(1 \cdot 0-1 \cdot 7)$ & $2 \cdot 3(1 \cdot 9-2 \cdot 9)$ & $1 \cdot 5(1 \cdot 2-1 \cdot 8)$ \\
\hline Quinolones & $7 \cdot 7(6 \cdot 3-9 \cdot 5)$ & $2 \cdot 4(1 \cdot 9-3 \cdot 0)$ & $1 \cdot 8(1 \cdot 4-2 \cdot 4)$ & $1 \cdot 5(1 \cdot 1-2 \cdot 2)$ & $1 \cdot 9(1 \cdot 5-2 \cdot 3)$ & $1 \cdot 1(0 \cdot 9-1 \cdot 4)$ \\
\hline Tetracyclines & $3 \cdot 9(2 \cdot 8-5 \cdot 3)$ & $1 \cdot 5(1 \cdot 1-2 \cdot 2)$ & $2 \cdot 6(1 \cdot 9-3 \cdot 6)$ & $1 \cdot 6(1 \cdot 1-2 \cdot 2)$ & $2 \cdot 4(1 \cdot 8-3 \cdot 2)$ & $1 \cdot 7(1 \cdot 3-2 \cdot 2)$ \\
\hline $\mathrm{TMP} / \mathrm{SMX}$ & $4 \cdot 8(3 \cdot 8-6 \cdot 1)$ & $1 \cdot 7(1 \cdot 3-2 \cdot 2)$ & $3 \cdot 5(2 \cdot 8-4 \cdot 5)$ & $2 \cdot 1(1 \cdot 6-2 \cdot 7)$ & $2 \cdot 2(1 \cdot 7-2 \cdot 7)$ & $1 \cdot 4(1 \cdot 1-1 \cdot 7)$ \\
\hline Vancomycin & $31 \cdot 1(15 \cdot 5-63)$ & $3 \cdot 3(1 \cdot 5-7 \cdot 2)$ & $0 \cdot 6(0 \cdot 1-2 \cdot 8)$ & $0 \cdot 2(0 \cdot 04-1 \cdot 0)$ & $2 \cdot 7(1 \cdot 2-6 \cdot 1)$ & $1 \cdot 1(0 \cdot 5-2 \cdot 6)$ \\
\hline \multicolumn{7}{|l|}{ Antibiotic prescription ${ }^{\mathrm{k}}$} \\
\hline 0 & Reference & Reference & Reference & Reference & Reference & Reference \\
\hline 1 & $1 \cdot 7(1 \cdot 5-2 \cdot 0)$ & $1 \cdot 7(1 \cdot 4-2 \cdot 0)$ & $1 \cdot 7(1 \cdot 5-2 \cdot 0)$ & $1 \cdot 7(1 \cdot 4-2 \cdot 0)$ & $1 \cdot 6(1.4-1 \cdot 8)$ & $1.5(1.4-1.7)$ \\
\hline $2-3$ & $3 \cdot 6(3 \cdot 0-4 \cdot 3)$ & $3 \cdot 6(3 \cdot 0-4 \cdot 4)$ & $2 \cdot 7(2 \cdot 3-3 \cdot 2)$ & $2 \cdot 5(2 \cdot 0-3 \cdot 0)$ & $2 \cdot 2(1 \cdot 9-2 \cdot 6)$ & $2 \cdot 1(1 \cdot 8-2 \cdot 5)$ \\
\hline$\geqslant 4$ & $9 \cdot 7(7 \cdot 7-12 \cdot 2)$ & $9 \cdot 0(7 \cdot 1-11 \cdot 4)$ & $3 \cdot 7(2 \cdot 9-4 \cdot 8)$ & $3 \cdot 7(2 \cdot 9-4 \cdot 8)$ & $2 \cdot 6(2 \cdot 1-3 \cdot 3)$ & $2 \cdot 4(1 \cdot 9-3 \cdot 1)$ \\
\hline
\end{tabular}

Data are no. (\%) of patients, unless otherwise indicated.

BMI, Body mass index; CI, confidence interval; CA-MRSA; community-associated methicillin-resistant S. aureus; HA-MRSA; healthcare-associated MRSA; OR, odds ratio; SSTI, skin and soft tissue infection; TMP/SMX, trimethoprim/sulfamethoxzole.

a SSTI cases and controls were frequency-matched to MRSA cases on age, sex, and year of diagnosis or outpatient encounter.

b Adjusted for category [ $<7,7$ to $<19,19$ to $<45,46$ to $<62$ (ref.), 62 to $<75$ and $\geqslant 75$ years), sex, race/ethnicity, ever-smoking status.

c White, non-Hispanic.

d Body mass index was categorized as normal $\left(<25 \mathrm{~kg} / \mathrm{m}^{2}\right)$, overweight $\left(25-29 \cdot 9 \mathrm{~kg} / \mathrm{m}^{2}\right)$ and obese $\left(\geqslant 30 \mathrm{~kg} / \mathrm{m}^{2}\right)$ for persons aged $18-59 \cdot 9$ years the most recent height and a weight within 2 years of the encounter/visit were used; for persons aged $\geqslant 60$ years the most recent height and weight within 1 year of encounter/visit were used. Missing either due to the absolute value of the $z$ score being $>5$ or if height and weight were not recorded in the vitals table within the 3 months before the diagnosis or visit.

e Body mass index $z$ scores for children aged 2-18 years were calculated using the 2000 CDC Growth Reference by implementing the zanthro function in Stata version 11 (normal, $z$ score $<85$ th percentile; overweight, 85th percentile $\leqslant z$ score $<95$ th percentile; obese, $z$ score $\geqslant 95$ th percentile.

f Season of onset: spring (March-May), summer (June-August), autumn (September-November), winter (December-February).

$\mathrm{g}$ The overall geocoding rate was $88.6 \%$, non-geocoding patients could not be assigned a community type or a community socioeconomic deprivation score and thus were omitted from multilevel analysis.

${ }^{\mathrm{h}}$ Census tracts were assigned to patients in cities due to the large geographical area and heterogeneous community of some cities.

i ORs for socioeconomic deprivation are quartile increase in level; a higher quartile represents a more deprived community.

j Order for an antimicrobial prescription in the 30-365 days before infection or visit, these are additionally adjusted for all other antibiotic classes in the table. Data on linezolid is not presented due to small cell sizes.

${ }^{k}$ Count of antimicrobial prescription order in the 30-365 days before infection or visit. 


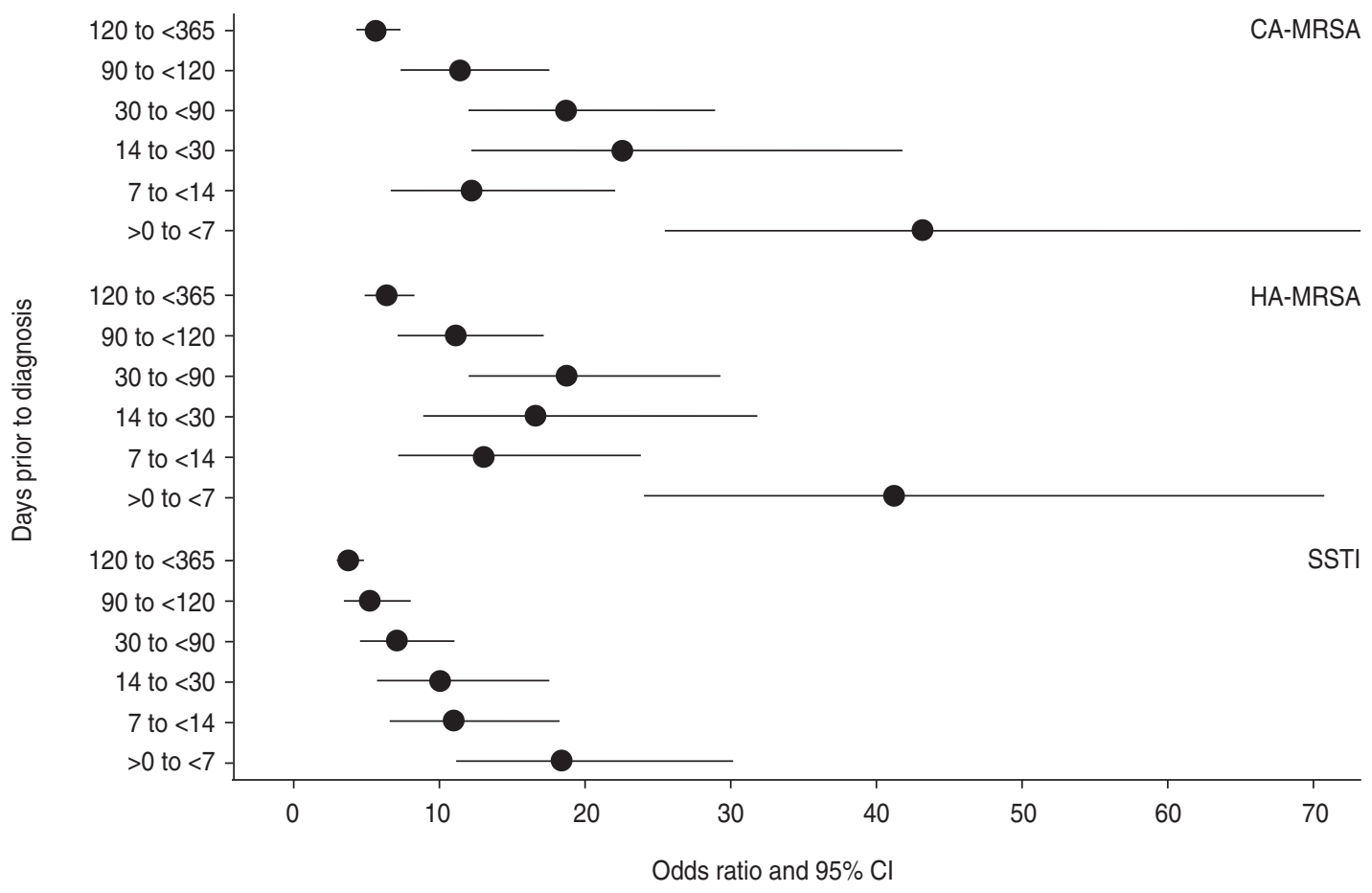

Fig. 3. Adjusted odds ratios for skin and soft tissue infection in the year preceding diagnosis, comparing CA- and HA-MRSA and SSTI cases to controls. SSTIs include: cellulitis and abscess, carbuncle and furuncle, erysipelas, impetigo, and unspecified local infection of skin and subcutaneous tissue. CA-MRSA, Community-associated MRSA; CI, confidence interval; HA-MRSA, healthcare-associated MRSA; SSTI, skin and soft tissue infection.

MRSA goes undiagnosed for an extended time or that compromised skin is a long-term risk factor for MRSA.

The results suggest that CA-MRSA cases were healthier than the other two infection groups and even controls, which could be due, in part, to the stringent CA-MRSA definition based on that of the CDC. For example, chronic kidney disease, diabetes, and some cardiac conditions were risk factors for HA-MRSA and SSTIs, but protective factors for CA-MRSA. This result may indicate that the CDC case definition for CA-MRSA may be too restrictive, leading to the study of a highly selected, very healthy group of patients due to misclassification of CA-MRSA as HA-MRSA cases. This misclassification may lead to underestimation of the burden of CA-MRSA infection.

Considering non-healthcare risk factors, obesity was a risk factor in paediatric patients for both HAand CA-MRSA, which to our knowledge has not been previously reported. Obesity was also associated with CA-MRSA infection in adults [30-32]. The literature is inconsistent regarding smoking as a risk for MRSA infection [20, 29, 30, 32]. Herein, cigarette smoking was identified as an independent risk factor for both HA- and CA-MRSA, even after adjustment for comorbidities and antibiotics. Summer and autumn were associated with increased odds of HA- and CA-MRSA consistent with previous studies $[4,11,33]$. CSD was associated with both HA-MRSA [34] and CA-MRSA infection. Residence in a city has been associated with MRSA infection [3, 15], attributed to injecting drug use (IDU) [25], crowding, and lower individual-level socioeconomic status [2]. Few previous studies have included non-urban areas [3, 4, 29]; their inclusion in the current study allowed us to identify increased risk in small towns compared to rural areas.

Consistent with previous studies, the majority of our CA-MRSA cases $[9,11,16,20,26,35]$ and many HA-MRSA cases presented with SSTIs, but HA-MRSA also presented with pneumonia, bacteraemia, endocarditis, and SSTIs, especially chronic skin ulcers and wound infections [6, 16, 25, 29, 35]. We also identified evidence of previous SSTI as a risk factor for subsequent MRSA infection, even controlling for antibiotic use. In the 30 days before diagnosis, this is probably due to the use of SSTI as a preliminary diagnosis in patients subsequently diagnosed with MRSA; however, as discussed 
Table 3. Associations of acute and chronic conditions in the 2 years preceding MRSA and SSTI with MRSA and SSTI case status compared to controls, in multinomial and binomial logistic regression models, respectively ${ }^{\mathrm{a}}$

\begin{tabular}{|c|c|c|c|c|c|c|c|c|c|c|c|c|}
\hline \multirow[b]{3}{*}{ Condition } & \multicolumn{6}{|c|}{ Unadjusted analysis $^{\mathrm{b}}$} & \multicolumn{6}{|c|}{ Adjusted analysis ${ }^{\mathrm{c}}$} \\
\hline & \multicolumn{2}{|l|}{ HA-MRSA } & \multicolumn{2}{|l|}{ CA-MRSA } & \multicolumn{2}{|l|}{ SSTI } & \multicolumn{2}{|l|}{ HA-MRSA } & \multicolumn{2}{|l|}{ CA-MRSA } & \multicolumn{2}{|l|}{ SSTI } \\
\hline & $\begin{array}{l}\text { Crude OR } \\
(95 \% \text { CI })\end{array}$ & $P$ & $\begin{array}{l}\text { Crude OR } \\
(95 \% \mathrm{CI})\end{array}$ & $P$ & $\begin{array}{l}\text { Crude OR } \\
(95 \% \mathrm{CI})\end{array}$ & $P$ & $\begin{array}{l}\text { Adjusted OR } \\
(95 \% \mathrm{CI})\end{array}$ & $P$ & $\begin{array}{l}\text { Adjusted OR } \\
(95 \% \mathrm{CI})\end{array}$ & $P$ & $\begin{array}{l}\text { Adjusted OR } \\
(95 \% \mathrm{CI})\end{array}$ & $P$ \\
\hline \multicolumn{13}{|l|}{ Respiratory tract } \\
\hline \multicolumn{13}{|l|}{ Acute } \\
\hline Bronchitis & $1 \cdot 9(1 \cdot 7-2 \cdot 3)$ & $<0 \cdot 001$ & $1 \cdot 4(1 \cdot 2-1 \cdot 6)$ & $<0.001$ & $1 \cdot 4(1 \cdot 3-1 \cdot 6)$ & $<0 \cdot 001$ & $1 \cdot 1(1 \cdot 0-1 \cdot 4)$ & $0 \cdot 14$ & $1 \cdot 1(0 \cdot 9-1 \cdot 3)$ & $0 \cdot 52$ & $1 \cdot 0(0 \cdot 9-1 \cdot 2)$ & $0 \cdot 96$ \\
\hline Otitis media & $0 \cdot 9(0 \cdot 7-1 \cdot 2)$ & 0.53 & $1 \cdot 9(1 \cdot 5-2 \cdot 3)$ & $<0.001$ & $1 \cdot 3(1 \cdot 0-1 \cdot 5)$ & $0 \cdot 01$ & $1 \cdot 0(0 \cdot 7-1 \cdot 3)$ & $0 \cdot 88$ & $1 \cdot 0(0 \cdot 8-1 \cdot 3)$ & $0 \cdot 97$ & $0 \cdot 9(0 \cdot 8-1 \cdot 1)$ & $0 \cdot 50$ \\
\hline Pharyngitis & $0.6(0 \cdot 5-0 \cdot 8)$ & $<0.001$ & $1 \cdot 5(1 \cdot 3-1 \cdot 8)$ & $<0.001$ & $1 \cdot 3(1 \cdot 2-1 \cdot 5)$ & $<0 \cdot 001$ & $0 \cdot 7(0 \cdot 6-0 \cdot 9)$ & $0 \cdot 01$ & $0 \cdot 8(0 \cdot 7-1 \cdot 0)$ & $0 \cdot 01$ & $1 \cdot 0(0 \cdot 9-1 \cdot 2)$ & $0 \cdot 60$ \\
\hline Rhinosinusitis & $0 \cdot 9(0 \cdot 7-1 \cdot 0)$ & $0 \cdot 08$ & $1 \cdot 5(1 \cdot 3-1 \cdot 7)$ & $<0.001$ & $1 \cdot 4(1 \cdot 3-1 \cdot 6)$ & $<0 \cdot 001$ & $0 \cdot 6(0 \cdot 5-0 \cdot 7)$ & $<0 \cdot 001$ & $0 \cdot 8(0 \cdot 7-1 \cdot 0)$ & $0 \cdot 01$ & $0 \cdot 9(0 \cdot 8-1 \cdot 1)$ & $0 \cdot 40$ \\
\hline Streptococcal sore throat & $0 \cdot 7(0 \cdot 4-1 \cdot 0)$ & $0 \cdot 07$ & $1 \cdot 8(1 \cdot 3-2 \cdot 5)$ & $<0 \cdot 001$ & $1 \cdot 4(1 \cdot 0-1 \cdot 8)$ & $0 \cdot 03$ & $0 \cdot 9(0 \cdot 6-1 \cdot 5)$ & $0 \cdot 81$ & $0 \cdot 9(0 \cdot 7-1 \cdot 3)$ & $0 \cdot 71$ & $1 \cdot 1(0 \cdot 8-1 \cdot 4)$ & $0 \cdot 72$ \\
\hline Upper respiratory infection & $1 \cdot 1(0 \cdot 9-1 \cdot 2)$ & $0 \cdot 54$ & $1 \cdot 6(1 \cdot 3-1 \cdot 8)$ & $<0 \cdot 001$ & $1 \cdot 3(1 \cdot 2-1 \cdot 5)$ & $<0 \cdot 001$ & $1 \cdot 1(0 \cdot 9-1 \cdot 3)$ & $0 \cdot 54$ & $1 \cdot 0(0 \cdot 8-1 \cdot 2)$ & $0 \cdot 92$ & $1 \cdot 1(1 \cdot 0-1 \cdot 3)$ & $0 \cdot 15$ \\
\hline \multicolumn{13}{|c|}{ Chronic } \\
\hline Asthma & $2 \cdot 1(1 \cdot 8-2 \cdot 6)$ & $<0.001$ & $1 \cdot 6(1 \cdot 3-1 \cdot 9)$ & $<0 \cdot 001$ & $1 \cdot 5(1 \cdot 3-1 \cdot 8)$ & $<0 \cdot 001$ & $1 \cdot 8(1 \cdot 5-2 \cdot 2)$ & $<0 \cdot 001$ & $1 \cdot 1(0 \cdot 9-1 \cdot 4)$ & $0 \cdot 21$ & $1 \cdot 2(1 \cdot 0-1 \cdot 4)$ & $0 \cdot 04$ \\
\hline COPD & $4 \cdot 0(3 \cdot 5-4 \cdot 6)$ & $<0 \cdot 001$ & $1 \cdot 1(1 \cdot 0-1 \cdot 3)$ & $0 \cdot 20$ & $1 \cdot 5(1 \cdot 4-1 \cdot 7)$ & $<0 \cdot 001$ & $2 \cdot 7(2 \cdot 3-3 \cdot 1)$ & $<0.001$ & $1 \cdot 0(0 \cdot 8-1 \cdot 1)$ & $0 \cdot 57$ & $1 \cdot 2(1 \cdot 1-1 \cdot 4)$ & $0 \cdot 002$ \\
\hline Lung diseases & $13 \cdot 7(11 \cdot 2-16 \cdot 8)$ & $<0.001$ & $0 \cdot 7(0 \cdot 5-1 \cdot 0)$ & $0 \cdot 05$ & $1 \cdot 6(1 \cdot 3-2 \cdot 0)$ & $<0.001$ & $8 \cdot 5(6 \cdot 8-10 \cdot 5)$ & $<0 \cdot 001$ & $0 \cdot 9(0 \cdot 6-1 \cdot 3)$ & $0 \cdot 52$ & $1 \cdot 2(0 \cdot 9-1 \cdot 5)$ & $0 \cdot 22$ \\
\hline Rhinosinusitis & $3 \cdot 2(2 \cdot 4-4 \cdot 4)$ & $<0.001$ & $1 \cdot 3(0.9-1.9)$ & $0 \cdot 19$ & $1 \cdot 6(1 \cdot 2-2 \cdot 2)$ & $0 \cdot 002$ & $2 \cdot 2(1 \cdot 6-3 \cdot 0)$ & $<0.001$ & $1 \cdot 0(0 \cdot 6-1 \cdot 4)$ & $0 \cdot 80$ & $1 \cdot 2(0 \cdot 9-1 \cdot 6)$ & $0 \cdot 24$ \\
\hline \multicolumn{13}{|l|}{ Cardiac } \\
\hline Heart diseases & $5 \cdot 6(4 \cdot 8-6 \cdot 5)$ & $<0.001$ & $0 \cdot 4(0 \cdot 3-0 \cdot 5)$ & $<0.001$ & $1 \cdot 3(1 \cdot 1-1 \cdot 5)$ & $<0.001$ & $3 \cdot 9(3 \cdot 2-4 \cdot 6)$ & $<0 \cdot 001$ & $0 \cdot 7(0 \cdot 5-0 \cdot 9)$ & $0 \cdot 01$ & $1 \cdot 2(1 \cdot 0-1 \cdot 4)$ & $0 \cdot 08$ \\
\hline Hypertension & $2 \cdot 7(2 \cdot 4-3 \cdot 1)$ & $<0.001$ & $0 \cdot 4(0 \cdot 4-0 \cdot 5)$ & $<0.001$ & $1 \cdot 1(1 \cdot 0-1 \cdot 3)$ & $0 \cdot 01$ & $1 \cdot 5(1 \cdot 3-1 \cdot 8)$ & $<0.001$ & $0.7(0.6-0.9)$ & $<0 \cdot 001$ & $1 \cdot 1(1 \cdot 0-1 \cdot 3)$ & $0 \cdot 11$ \\
\hline Lipid disorders & $1 \cdot 9(1 \cdot 7-2 \cdot 2)$ & $<0.001$ & $0.5(0.4-0.6)$ & $<0.001$ & $1 \cdot 2(1 \cdot 1-1 \cdot 3)$ & $<0 \cdot 001$ & $0 \cdot 9(0 \cdot 8-1 \cdot 1)$ & $0 \cdot 27$ & $0.7(0.6-0.9)$ & $<0 \cdot 001$ & $1 \cdot 2(1 \cdot 1-1 \cdot 4)$ & $0 \cdot 002$ \\
\hline \multicolumn{13}{|l|}{ Skin and soft tissue } \\
\hline Carbuncle/furuncle ${ }^{\mathrm{d}}$ & $5 \cdot 9(4 \cdot 0-8 \cdot 8)$ & $<0.001$ & $10 \cdot 1(7 \cdot 0-14 \cdot 6)$ & $<0.001$ & $3 \cdot 8(2 \cdot 6-5 \cdot 6)$ & $<0 \cdot 001$ & $4 \cdot 7(3 \cdot 1-7 \cdot 0)$ & $<0 \cdot 001$ & $6 \cdot 8(4 \cdot 7-10 \cdot 0)$ & $<0.001$ & $2 \cdot 5(2 \cdot 1-3 \cdot 0)$ & $<0 \cdot 001$ \\
\hline Cellulitis/abscess ${ }^{\mathrm{d}}$ & $5 \cdot 6(4 \cdot 6-6 \cdot 8)$ & $<0 \cdot 001$ & $3 \cdot 4(2 \cdot 8-4 \cdot 2)$ & $<0.001$ & $3 \cdot 1(2 \cdot 6-3 \cdot 8)$ & $<0 \cdot 001$ & $3 \cdot 5(2 \cdot 8-4 \cdot 3)$ & $<0.001$ & $3 \cdot 0(2 \cdot 4-3 \cdot 7)$ & $<0 \cdot 001$ & $2 \cdot 7(1 \cdot 8-3 \cdot 9)$ & $<0 \cdot 001$ \\
\hline Chronic ulcer of skin & $42 \cdot 3(26 \cdot 5-68)$ & $<0.001$ & $4 \cdot 4(2 \cdot 5-7 \cdot 6)$ & $<0.001$ & $2 \cdot 9(1.7-4.9)$ & $<0.001$ & $26 \cdot 9(16 \cdot 7-43 \cdot 4)$ & $<0.001$ & $6 \cdot 7(2 \cdot 1-2 \cdot 7)$ & $<0 \cdot 001$ & $2 \cdot 1(1 \cdot 3-3 \cdot 7)$ & $0 \cdot 01$ \\
\hline \multicolumn{13}{|l|}{ Other } \\
\hline Chronic kidney disease & $6 \cdot 3(5 \cdot 0-8 \cdot 0)$ & $<0 \cdot 001$ & $0 \cdot 2(0 \cdot 1-0 \cdot 4)$ & $<0.001$ & $1 \cdot 6(1 \cdot 3-2 \cdot 1)$ & $<0 \cdot 001$ & $4 \cdot 1(3 \cdot 1-5 \cdot 3)$ & $<0 \cdot 001$ & $0 \cdot 4(0 \cdot 2-0 \cdot 8)$ & $0 \cdot 01$ & $1 \cdot 5(1 \cdot 1-1 \cdot 9)$ & $0 \cdot 01$ \\
\hline Diabetes & $4 \cdot 0(3 \cdot 4-4 \cdot 6)$ & $<0.001$ & $0.6(0.5-0.8)$ & $<0 \cdot 001$ & $1.6(1.4-1.9)$ & $<0 \cdot 001$ & $2 \cdot 6(2 \cdot 2-3 \cdot 1)$ & $<0.001$ & $1 \cdot 0(0 \cdot 8-1 \cdot 3)$ & $0 \cdot 82$ & $1 \cdot 5(1 \cdot 3-1 \cdot 8)$ & $<0.001$ \\
\hline
\end{tabular}

CA-MRSA, Community-associated methicillin-resistant S. aureus; COPD, chronic obstructive pulmonary disease; CI, confidence interval; HA-MRSA; healthcareassociated MRSA; OR, odds ratio; SSTI, skin and soft tissue infection.

a SSTI cases and controls were frequency-matched to MRSA cases on age, sex, and year of diagnosis or outpatient encounter.

$\mathrm{b}$ Models the association between the condition identified in inpatient, outpatient, or emergency department encounters, or on problem list or in medications table (excluding antibiotic prescriptions) in the 2 years preceding diagnosis or visit and case status.

c Adjusted for age category [ $<7,7$ to $<19,19$ to $<45,46$ to $<62$ (ref.), 62 to $<75$ and $\geqslant 75$ years], sex, race/ethnicity, ever-smoking status, and any antibiotic order in the previous 2 years.

$\mathrm{d}$ The 30 days preceding infection or visit were excluded for carbuncle, furuncle, cellulitis or abscess to avoid protopathic bias. 
Table 4. Adjusted associations of antibiotic orders for acute and chronic conditions in the 2 years preceding MRSA and SSTI, compared to controls, in multinomial and binomial logistic regression models, respectively

\begin{tabular}{|c|c|c|c|c|c|c|}
\hline \multirow[b]{2}{*}{ Condition } & \multicolumn{2}{|l|}{ HA-MRSA } & \multicolumn{2}{|l|}{ CA-MRSA } & \multicolumn{2}{|l|}{ SSTI } \\
\hline & $\begin{array}{l}\text { Adjusted OR }{ }^{\mathrm{a}} \\
(95 \% \mathrm{CI})\end{array}$ & $P$ & $\begin{array}{l}\text { Adjusted OR } \\
(95 \% \mathrm{CI})\end{array}$ & $P$ & $\begin{array}{l}\text { Adjusted OR } \\
(95 \% \mathrm{CI})\end{array}$ & $P$ \\
\hline \multicolumn{7}{|l|}{ Respiratory tract } \\
\hline \multicolumn{7}{|l|}{ Acute } \\
\hline Bronchitis & $1 \cdot 3(1 \cdot 1-1 \cdot 6)$ & $0 \cdot 003$ & $1 \cdot 3(1 \cdot 1-1 \cdot 6)$ & $0 \cdot 004$ & $1 \cdot 3(1 \cdot 1-1 \cdot 5)$ & $<0.001$ \\
\hline Otitis media & $1 \cdot 4(1 \cdot 0-2 \cdot 0)$ & $0 \cdot 03$ & $1 \cdot 4(1 \cdot 1-1 \cdot 8)$ & $0 \cdot 004$ & $1 \cdot 3(1 \cdot 0-1 \cdot 6)$ & $0 \cdot 06$ \\
\hline Pharyngitis & $1 \cdot 2(0 \cdot 9-1 \cdot 7)$ & $0 \cdot 28$ & $1 \cdot 0(0 \cdot 7-1 \cdot 3)$ & $0 \cdot 83$ & $1 \cdot 0(0 \cdot 8-1 \cdot 3)$ & $0 \cdot 71$ \\
\hline Rhinosinusitis & $1 \cdot 0(0 \cdot 8-1 \cdot 1)$ & $0 \cdot 66$ & $1 \cdot 3(1 \cdot 1-1 \cdot 5)$ & $<0.001$ & $1 \cdot 4(1 \cdot 2-1 \cdot 5)$ & $<0 \cdot 001$ \\
\hline Streptococcal sore throat & $1 \cdot 1(0 \cdot 7-1 \cdot 9)$ & $0 \cdot 70$ & $1 \cdot 2(0 \cdot 9-1 \cdot 7)$ & $0 \cdot 29$ & $1 \cdot 5(1 \cdot 1-2 \cdot 0)$ & $0 \cdot 01$ \\
\hline Upper respiratory infection & $1 \cdot 7(1 \cdot 2-2 \cdot 4)$ & $0 \cdot 002$ & $1 \cdot 1(0 \cdot 8-1 \cdot 6)$ & $0 \cdot 54$ & $1 \cdot 4(1 \cdot 0-1 \cdot 8)$ & $0 \cdot 01$ \\
\hline \multicolumn{7}{|l|}{ Chronic } \\
\hline Asthma & $4 \cdot 3(2 \cdot 2-8 \cdot 2)$ & $<0.001$ & $1 \cdot 4(0 \cdot 6-3 \cdot 1)$ & $0 \cdot 39$ & $2 \cdot 1(1 \cdot 2-4 \cdot 0)$ & $0 \cdot 01$ \\
\hline COPD & $3 \cdot 2(2 \cdot 2-4 \cdot 6)$ & $<0.001$ & $1 \cdot 6(1 \cdot 1-2 \cdot 4)$ & $0 \cdot 02$ & $1 \cdot 5(1 \cdot 1-2 \cdot 2)$ & $0 \cdot 01$ \\
\hline Lung diseases & $2 \cdot 6(1 \cdot 6-4 \cdot 4)$ & $<0 \cdot 001$ & $1 \cdot 7(0 \cdot 9-3 \cdot 6)$ & $0 \cdot 12$ & $1 \cdot 1(0 \cdot 6-1 \cdot 9)$ & $0 \cdot 18$ \\
\hline Rhinosinusitis & $1 \cdot 5(0 \cdot 9-2 \cdot 7)$ & $0 \cdot 13$ & $1 \cdot 1(0 \cdot 6-2 \cdot 0)$ & $0 \cdot 65$ & $1 \cdot 1(0 \cdot 6-1 \cdot 7)$ & $0 \cdot 63$ \\
\hline \multicolumn{7}{|l|}{ Skin and soft tissue } \\
\hline Carbuncle/furuncle ${ }^{\mathrm{b}}$ & $8 \cdot 9(5 \cdot 7-14 \cdot 0)$ & $<0 \cdot 001$ & $17 \cdot 9(11 \cdot 8-27)$ & $<0 \cdot 001$ & $4 \cdot 7(3 \cdot 0-7 \cdot 2)$ & $<0.001$ \\
\hline Cellulitis/abscess ${ }^{\mathrm{b}}$ & $5 \cdot 2(4 \cdot 2-6 \cdot 6)$ & $<0.001$ & $6 \cdot 7(5 \cdot 4-8 \cdot 3)$ & $<0 \cdot 001$ & $4 \cdot 2(3 \cdot 4-5 \cdot 2)$ & $<0.001$ \\
\hline Chronic ulcer of skin & $16 \cdot 6(7 \cdot 1-38 \cdot 8)$ & $<0 \cdot 001$ & $6 \cdot 5(2 \cdot 4-17 \cdot 4)$ & $<0 \cdot 001$ & $3 \cdot 4(1 \cdot 4-8 \cdot 4)$ & $0 \cdot 003$ \\
\hline
\end{tabular}

CA-MRSA, Community-associated methicillin-resistant $S$. aureus; COPD, chronic obstructive pulmonary disease; CI, confidence interval; HA-MRSA; healthcare-associated MRSA; OR, odds ratio; SSTI, skin and soft tissue infection.

a Models the association between any antibiotic order for the condition in the 2 years preceding diagnosis or visit and case status; adjusted for age category ( $<7,7$ to $<19,19$ to $<45,46$ to $<62$ (ref.), 62 to $<75$ and $\geqslant 75$ years], sex, race/ethnicity, ever-smoking status.

b The 30 days preceding infection or visit were excluded for carbuncle, furuncle, cellulitis or abscess to avoid protopathic bias.

previously, the association remained strong in all earlier time windows out to 1 year before MRSA diagnosis.

Our data suggest that previous antibiotic use is a risk factor for MRSA in both sexes and across the age range. Although often assumed, this relationship has rarely been rigorously assessed [36, 37]. In accord with two English studies, the relationship between antibiotic use and MRSA varied by antibiotic class and number of times ordered $[14,30]$. No conditions, other than SSTI, were significantly associated with CA-MRSA after accounting for antibiotic administration. There were many antibiotics administered for conditions that are usually caused by viruses (e.g. bronchitis, rhinosinusitis, otitis media) and there was risk of MRSA associated with antibiotic prescriptions for those conditions, providing additional evidence supporting more judicious antibiotic use in the outpatient setting.

MRSA infection may go undiagnosed since previous studies have suggested that empirical treatment or incision and drainage is often sufficient to treat CA-MRSA SSTI [38, 39]. We included a SSTI case group to assess this possibility. As anticipated, similar associations were observed in the SSTI and CAMRSA groups. We also found COPD, diabetes and skin conditions associated with SSTI, consistent with known risk factors for SSTI [40]. Examination of the similarities and differences in risk factor associations with HA-MRSA, CA-MRSA and SSTIs may support the conclusion that there is undiagnosed MRSA in the SSTI cases.

The strengths of this study include the wealth of longitudinal clinical data available for both paediatric and adult patients. We used two methods to identify MRSA cases with high sensitivity and subgroup analysis that required MRSA cases to have both a positive culture and an ICD-9 code confirming the reported associations. The EHR allowed the careful application of criteria for CA-MRSA classification, identification of patients with SSTIs that might represent undiagnosed MRSA cases, as well as 
comprehensive adjusted analysis, including consideration of antibiotic administration.

This study also had limitations. Molecular typing of isolates was not completed and culture data was not available for every MRSA case, consistent with practice in the community. The EHR did not have information on individual-level socioeconomic status and other CA-MRSA risk factors, such as crowded living conditions, incarceration, IDU, and athlete $[1,2,27,41]$. It should be noted that adjustment for place-level CSD did not lead to substantive changes in associations.

This study has several important implications. CA-MRSA incidence in a general US population continues to rise, as does SSTI incidence, but less markedly. We identified several opportunities for intervention by identifying modifiable risk factors for CA-MRSA including avoidance of unnecessary antimicrobial prescribing particularly in the setting of upper respiratory tract infection. Finally, the study demonstrates the utility of EHR data for epidemiological research, a practice likely to increase in the future due to incentives provided by the Patient Protection and Affordable Healthcare Act.

\section{ACKNOWLEDGEMENTS}

This work was supported by the Center for a Livable Future at Johns Hopkins Bloomberg School of Public Health, the Seed Grant Program for Joint Research of New York University School of Medicine and the Geisinger Health System (B.S.), and the National Institute of Environmental Health Sciences (J.C., Training Grant ES07141).

\section{DECLARATION OF INTEREST}

None.

\section{REFERENCES}

1. Skov RL, Jensen KS. Community-associated methicillin-resistant Staphylococcus aureus as a cause of hospital-acquired infections. Journal of Hospital Infection 2009; 73: 364-370.

2. David MZ, Daum RS. Community-associated methicillin-resistant Staphylococcus aureus: epidemiology and clinical consequences of an emerging epidemic. Clinical Microbiology Reviews 2010; 23: 616-687.

3. Morin CA, Hadler JL. Population-based incidence and characteristics of community-onset Staphylococcus aureus infections with bacteremia in 4 metropolitan
Connecticut areas, 1998. Journal of Infectious Diseases 2001; 184: 1029-1034.

4. Van De Griend $\mathbf{P}$, et al. Community-associated methicillin-resistant Staphylococcus aureus, Iowa, USA. Emerging Infectious Diseases 2009; 15: 1582-1589.

5. El Atrouni WI, et al. Temporal trends in the incidence of Staphylococcus aureus bacteremia in Olmsted County, Minnesota, 1998 to 2005: a populationbased study. Clinical Infectious Diseases 2009; 49: e130-138.

6. Kallen AJ, et al. Health care-associated invasive MRSA infections, 2005-2008. The Journal of the American Medical Association 2010; 304: 641-648.

7. Lessa FC, et al. Comparison of incidence of bloodstream infection with methicillin-resistant Staphylococcus aureus between England and United States, 2006-2007. Clinical Infectious Diseases 2010; 51: 925-928.

8. Tracy LA, et al. Staphylococcus aureus infections in US veterans, Maryland, USA, 1999-2008. Emerging Infectious Diseases 2011; 17: 441-448.

9. Buss BF, et al. Population-based estimates of methicillin-resistant Staphylococcus aureus (MRSA) infections among high school athletes-Nebraska, 2006-2008. Journal of School Nursing 2009; 25: 282-291.

10. Caffrey AR, Laplante KL. Changing epidemiology of methicillin-resistant Staphylococcus aureus in the Veterans Affairs Healthcare System, 2002-2009. Infection. Published online: 13 December 2011. doi:10.1007/s15010-011-0232-3.

11. Crum NF, et al. Fifteen-year study of the changing epidemiology of methicillin-resistant Staphylococcus aureus. American Journal of Medicine 2006; 119: 943-951.

12. Frei CR, et al. Emergence of community-acquired methicillin-resistant Staphylococcus aureus skin and soft tissue infections as a common cause of hospitalization in United States children. Journal of Pediatric Surgery 2010; 45: 1967-1974.

13. Li F, Miller FD, Effler PV. Epidemiology of methicillinresistant Staphylococcus aureus among incarcerated population in Hawai'i, 2000-2005. Hawai'i Journal of Medicine \& Public Health 2010; 69: 99-102.

14. Schneider-Lindner V, et al. Antibacterial drugs and the risk of community-associated methicillin-resistant Staphylococcus aureus in children. Archives of Pediatrics and Adolescent Medicine 2011; 165: 1107-1114.

15. Klevens RM, et al. Invasive methicillin-resistant Staphylococcus aureus infections in the United States. Journal of the American Medical Association 2007; 298 : 1763-1771.

16. Liu C, et al. A population-based study of the incidence and molecular epidemiology of methicillin-resistant Staphylococcus aureus disease in San Francisco, 2004-2005. Clinical Infectious Diseases 2008; 46: 1637 1646.

17. Klein E, Smith DL, Laxminarayan R. Communityassociated methicillin-resistant Staphylococcus aureus in outpatients, United States, 1999-2006. Emerging Infectious Diseases 2009; 15: 1925-1930. 
18. Mera RM, et al. Increasing role of Staphylococcus aureus and community-acquired methicillin-resistant Staphylococcus aureus infections in the United States: a 10-year trend of replacement and expansion. Microbial Drug Resistance 2011; 17: 321-328.

19. Farr AM, et al. Trends in hospitalization for community-associated methicillin-resistant Staphylococcus aureus in New York City, 1997-2006: data from New York State's Statewide Planning and Research Cooperative System. Infection Control and Hospital Epidemiology 2012; 33: 725-731.

20. Fridkin SK, et al. Methicillin-resistant Staphylococcus aureus disease in three communities. New England Journal of Medicine 2005; 352: 1436-1444.

21. Schwartz BS, et al. Body Mass Index and the Built and Social Environments in Children and Adolescents Using Electronic Health Records. American Journal of Preventive Medicine 2011; 41: e17-e28.

22. Morrison MA, Hageman JC, Klevens RM. Case definition for community-associated methicillin-resistant Staphylococcus aureus. Journal of Hospital Infection 2006; 62: 241.

23. Liu AY, et al. Associations of the burden of coal abandoned mine lands with three dimensions of community context in Pennsylvania. ISRN Public Health 2012; 2012: 11 .

24. Feinstein AR, Horwitz RI. An algebraic analysis of biases due to exclusion, susceptibility, and protopathic prescription in case-control research. Journal of Chronic Diseases 1981; 34: 393-403.

25. Huang $\mathbf{H}$, et al. Comparisons of community-associated methicillin-resistant Staphylococcus aureus (MRSA) and hospital-associated MSRA infections in Sacramento, California. Journal of Clinical Microbiology 2006; 44: 2423-2427.

26. Kim J, et al. Changing epidemiology of methicillinresistant Staphylococcus aureus in Alberta, Canada: population-based surveillance, 2005-2008. Epidemiology and Infection 2011; 139: 1009-1018.

27. Klevens RM, et al. Community-associated methicillinresistant Staphylococcus aureus and healthcare risk factors. Emerging Infectious Diseases 2006; 12: 19911993.

28. Kuehnert MJ, et al. Methicillin-resistant Staphylococcus aureus hospitalizations, United States. Emerging Infectious Diseases 2005; 11: 868-872.

29. Naimi TS, et al. Comparison of community- and health care-associated methicillin-resistant Staphylococcus aureus infection. Journal of the American Medical Association 2003; 290: 2976-2984.

30. Schneider-Lindner V, et al. Antimicrobial drugs and community-acquired methicillin-resistant Staphy- lococcus aureus, United Kingdom. Emerging Infectious Diseases 2007; 13: 994-1000.

31. Methicillin-resistant Staphylococcus aureus among players on a high school football team - New York City, 2007. Morbidity and Mortality Weekly Report 2009; 58: 52-55.

32. Khawcharoenporn T, et al. Risk factors for communityassociated methicillin-resistant Staphylococcus aureus cellulitis-and the value of recognition. Hawai'i Journal of Medicine and Public Health 2010; 69: 232-236.

33. Morrison-Rodriguez SM, et al. Community-associated methicillin-resistant Staphylococcus aureus infections at an Army training installation. Epidemiology and Infection 2010; 138: 721-729.

34. Bagger JP, Zindrou D, Taylor KM. Postoperative infection with meticillin-resistant Staphylococcus aureus and socioeconomic background. Lancet 2004; 363: 706-708.

35. Larsen AR, et al. Emergence and characterization of community-associated methicillin-resistant Staphyloccocus aureus infections in Denmark, 1999 to 2006. Journal of Clinical Microbiology 2009; 47: 73-78.

36. Skiest DJ, et al. Prospective comparison of methicillinsusceptible and methicillin-resistant communityassociated Staphylococcus aureus infections in hospitalized patients. Journal of Infection 2007; 54: 427-434.

37. Como-Sabetti KJ, et al. Risk factors for communityassociated Staphylococcus aureus infections: results from parallel studies including methicillin-resistant and methicillin-sensitive $S$. aureus compared to uninfected controls. Epidemiology and Infection 2011; 139: 419429.

38. Hankin A, Everett WW. Are antibiotics necessary after incision and drainage of a cutaneous abscess? Annals of Emergency Medicine 2007; 50: 49-51.

39. Rajendran PM, et al. Randomized, double-blind, placebo-controlled trial of cephalexin for treatment of uncomplicated skin abscesses in a population at risk for community-acquired methicillin-resistant Staphylococcus aureus infection. Antimicrobial Agents and Chemotherapy $2007 ; \mathbf{5 1}$ : 4044-4048.

40. Tognetti L, et al. Bacterial skin and soft tissue infections: review of the epidemiology, microbiology, aetiopathogenesis and treatment: A collaboration between dermatologists and infectivologists. Journal of the European Academy of Dermatology and Venereology. Published online: 7 January 2012. doi:10.1111/j.14683083.2011.04416.x.

41. Salgado CD, Farr BM, Calfee DP. Communityacquired methicillin-resistant Staphylococcus aureus: a meta-analysis of prevalence and risk factors. Clinical Infectious Diseases 2003; 36: 131-139. 